\title{
The Ratio of High-Molecular Weight Adiponectin and Total Adiponectin Differs in Preterm and Term Infants
}

\author{
TOMOHIDE YOSHIDA, HIRAKU NAGASAKI, YOSHIHIDE ASATO, AND TAKAO OHTA
}

Department of Pediatrics, University of the Ryukyus, Nishihara, Okinawa 903-0125, Japan

\begin{abstract}
Adiponectin consists of three subspecies (high-, middleand low-molecular weight adiponectin). Among these, high-molecular weight adiponectin (H-adn) is suggested to be an active form of this protein. To assess the relationship between $\mathrm{H}$-adn and postnatal growth in preterm infants (PIs), serum H-adn and total adiponectin (T-adn) were measured in 46 PIs at birth and at corrected term, and 26 term infants (TI) at birth. T-adn and $\mathrm{H}$-adn concentrations, and the ratio of $\mathrm{H}$-adn to $\mathrm{T}$-adn (H/T-adn) were significantly greater in TI and PI at corrected term than in PI at birth $(p<0.001)$. T-adn and $\mathrm{H}$-adn concentrations in PI at corrected term were similar to those in TI, but H/T-adn in PI at corrected term was less than that in TI $(p<0.02)$. Stepwise multiple regression analysis revealed that the factors contributing to H/T-adn and serum concentrations of $\mathrm{T}$ - and $\mathrm{H}$-adn in PI at corrected term were different from those in TI. These data suggest that quality of early postnatal growth in PIs is different from that in normally developed TI. Postnatal growth accompanying adipose tissue similar to TI may be important for PI to prevent future development of cardiovascular disease. (Pediatr Res 65: 580-583, 2009)
\end{abstract}

$\mathrm{E}^{\mathrm{p}}$ pidemiologic studies have demonstrated that low birth weight is associated with an increased risk for atherosclerotic cardiovascular disease (CVD) later in life (1-3); however, the mechanisms behind this relationship have yet to be fully elucidated. Adiponectin is one of several adipocytokines secreted by the adipocytes (4) and has numerous physiologic functions. Serum levels of adiponectin are inversely associated with insulin resistance, inflammatory markers, and CVD risk factors (5-7). Taken together, these data suggest that adiponectin has antidiabetic, antiinflammatory, and antiatherogenic functions. Adiponectin consists of three multimer species (high-, middle-, and low-molecular weight adiponectins: H-adn, M-adn, and L-adn) (8-11). Among these, $\mathrm{H}$-adn is suggested to be an active form of this protein, having a higher bound affinity to surface of the cell membrane and remarkably high adenosine monophosphate kinase activation compared with M-adn and L-adn (10,11). Furthermore, the ratio of $\mathrm{H}$-adn and total adiponectin (T-adn) (H/Tadn) are more significantly associated with insulin resistance than T-adn, thereby suggesting the usefulness of the H/T-adn in diagnosing insulin resistance (12).

Serum levels of adiponectin are inversely associated with body weight and body mass index (BMI) in adults and schoolchildren $(4,13)$. In contrast, serum levels of adiponectin in

Received September 23, 2008; accepted November 26, 2008.

Correspondence: Takao Ohta, M.D., Ph.D., Department of Pediatrics, Faculty of Medicine, University of the Ryukyus, 207 Uehara, Nishihara, Okinawa 903-0125, Japan; e-mail: tohta@med.u-ryukyu.ac.jp

Supported by Health Sciences Research Grants (Research on Specific Diseases) from the Ministry of Health, Labour, and Welfare, Japan, and by a Grant-in-aid for Scientific Research (B: 17390303) from the Ministry of Education, Culture, Sports, Science and Technology, Japan. neonates were positively associated with fetal birth weight (14), with their levels being much higher than those in adults and schoolchildren $(2,4,7,13,14)$. The physiologic significance of these findings remains to be clarified, and no information exists regarding serum multimeric adiponectin concentrations in the fetal and neonatal period. Thus, in the present study, as a first step in clarifying the relationship between low birth weight and future CVD risk, we investigated the serum concentrations of multimeric adiponectin in neonates and the difference between premature and mature infants.

\section{SUBJECTS AND METHODS}

The subjects consisted of 72 newborn infants ( 46 preterm born between weeks 24 and 35 of gestation and 26 term and near-term infants born after week 36 of gestation from an uncomplicated pregnancy) who had been admitted to the Ryukyu University hospital, Japan. All term and near-term infants were appropriate for gestational age (AGA). In preterm infants (PIs), 37 infants were AGA and nine infants were small for gestational age (SGA). Preterm deliveries were induced because of threatened premature delivery (21 infants), nonreassuring fetal status (four infants), viral infection of mother (one infant), premature rupture of membrane (13 infants), placenta previa (four infants), hypertension of mother (two infants), and cervical cancer of mother (one infant). Among 46 mothers, two mothers had pregnancy-induced hypertension. All PIs were fed breast milk and infant formula. Serum sample collection and anthropometric measurements were performed at birth and at the corrected term of PIs. Written informed consent was obtained from the parents. The Ethics Committee of the Ryukyu University approved the study protocol.

Anthropometric measurements of subjects. Gestational age was confirmed by ultrasound before week 20 of gestation. The umbilical cord was cut, and the placental weight was measured with a calibrated scale and recorded. The infants were weighed immediately after birth, and birth length was measured using a measuring board. Heart rate (HR) and blood pressure in the lower limb were measured with an automated oscillometric device (MP-60, Phillips Medical Systems, Eindhoven, The Netherlands). Neonatal BMI and Ponderal index were calculated as weight $(\mathrm{kg}) /$ body length ${ }^{2}\left(\mathrm{~m}^{2}\right)$ and weight $(\mathrm{kg}) /$ body length ${ }^{3}\left(\mathrm{~m}^{3}\right)$, respectively.

Laboratory measurements. Umbilical vein samples were drawn and samples were stored at $-40^{\circ} \mathrm{C}$ until assay. Serum adiponectin concentrations were measured by sandwich ELISA kits (Daiichi Pure Chemicals Co. Ltd., Tokyo, Japan) with a dynamic range of $0.075-4.8 \mathrm{ng} / \mathrm{mL}$. Intraassay variations $(\mathrm{CV})$ were $5.3 \%$ (T-Adn), $4.1 \%$ (M-adn $+\mathrm{H}$-adn), and 3.3\% ( $\mathrm{H}$-adn), as described previously (15).

Statistical evaluation. The differences between series of data were determined by Wilcoxon's rank sum test. Levels of T-adn, H-adn, and H/T-adn were markedly skewed. Thus, these parameters were normalized by $\log$ transformation. Pearson's correlation coefficient test was performed to assess the associations between data. To examine the relationship between adiponectin multimeric complexes and clinical data, a forward stepwise multiple regression analysis was performed. Values are reported as the mean $\pm \mathrm{SD}$. Significance was set at $p<0.05$. All analyses were performed with JMP 5.1 (SAS Institute Inc., Cary, NC).

Abbreviations: adn, adiponectin, CVD, cardiovascular disease, DBP, diastolic blood pressure, H-adn, high-molecular weight adiponectin, H/T-adn, the ratio of high-molecular weight adiponectin to total adiponectin, HR, heart rate, PI, preterm infant, SBP, systolic blood pressure, T-adn, total adiponectin, TI, term infants 
Table 1. Clinical and chemical data on preterm and term infants

\begin{tabular}{|c|c|c|c|c|c|}
\hline & \multicolumn{4}{|c|}{ Preterm infants } & \multirow[b]{2}{*}{ Term infants } \\
\hline & At birth & $p$ & At corrected term & $p$ & \\
\hline Number of subjects $(\mathrm{M} / \mathrm{F})$ & $46(19 / 28)$ & & & & $26(11 / 15)$ \\
\hline Postmenstrual age (wk) & $31.7 \pm 2.9$ & $<0.001$ & $37.1 \pm 1.1$ & ns & $37.7 \pm 1.2 *$ \\
\hline Placental weight (g) & $371 \pm 102$ & & & & $535 \pm 138 *$ \\
\hline Body weight (g) & $1609 \pm 492$ & $<0.001$ & $2229 \pm 374$ & $<0.001$ & $2908 \pm 574^{*}$ \\
\hline Body length (cm) & $40.9 \pm 4.2$ & $<0.001$ & $43.8 \pm 2.7$ & $<0.001$ & $47.3 \pm 2.1^{*}$ \\
\hline BMI $\left(\mathrm{kg} / \mathrm{m}^{2}\right)$ & $9.3 \pm 1.5$ & $<0.001$ & $11.6 \pm 1.5$ & $<0.001$ & $12.9 \pm 1.7^{*}$ \\
\hline Ponderal index $\left(\mathrm{kg} / \mathrm{m}^{3}\right)$ & $2.27 \pm 0.29$ & $<0.001$ & $2.69 \pm 0.42$ & ns & $2.74 \pm 0.34 *$ \\
\hline $\mathrm{SBP}(\mathrm{mm} \mathrm{Hg})$ & $61 \pm 8$ & $<0.001$ & $69 \pm 9$ & $<0.001$ & $62 \pm 5$ \\
\hline DBP (mm Hg) & $35 \pm 7$ & ns & $36 \pm 7$ & ns & $36 \pm 7$ \\
\hline Heart rate & $134 \pm 12$ & $<0.01$ & $144 \pm 12$ & $<0.001$ & $123 \pm 17 \dagger$ \\
\hline $\mathrm{T}-\operatorname{adn}(\mu \mathrm{g} / \mathrm{mL})$ & $6.2 \pm 3.3$ & $<0.001$ & $19.9 \pm 13.5$ & ns & $17.0 \pm 10.1^{*}$ \\
\hline $\mathrm{H}-\mathrm{adn}(\mu \mathrm{g} / \mathrm{mL})$ & $2.6 \pm 2.0$ & $<0.001$ & $9.6 \pm 5.7$ & ns & $9.9 \pm 6.0^{*}$ \\
\hline M-adn $(\mu \mathrm{g} / \mathrm{mL})$ & $1.5 \pm 1.0$ & $<0.001$ & $5.3 \pm 5.2$ & ns & $3.8 \pm 3.5 \dagger$ \\
\hline L-adn $(\mu \mathrm{g} / \mathrm{mL})$ & $2.1 \pm 1.2$ & $<0.01$ & $5.0 \pm 6.7$ & ns & $3.3 \pm 2.1$ \\
\hline $\mathrm{H} / \mathrm{T}$-adn & $0.37 \pm 0.16$ & $<0.001$ & $0.50 \pm 0.12$ & $<0.02$ & $0.58 \pm 0.11^{*}$ \\
\hline
\end{tabular}

$* p<0.001$, significantly different from preterm infants at birth.

$\dagger p<0.05$, significantly different from preterm infants at birth.

$\mathrm{M}$, male; F, female; ns, not significant.

\section{RESULTS}

All data presented were not changed even after removing data of SGA infants. Therefore, we did not separate the data for SGA and AGA in analysis.

Characteristics of the subjects. As shown in Table 1, postmenstrual age of PI was significantly younger than that of term infant (TI). Postmenstrual age at corrected term of PI was similar to that of TI. Placental weight was significantly lighter in PI than that in TI. Body sizes (weight and length) were much smaller in PI at birth than those in TI. Body sizes were significantly larger in PI at corrected term than those at birth but still significantly smaller than those in TI. Systolic blood pressure (SBP) of PI at birth was similar to that of TI. SBP of PI at corrected term was significantly higher than those of PI at birth and TI. No differences in diastolic blood pressure (DBP) were found among the three groups. HR was significantly greater in PI at corrected term than in PI at birth and in TI. HR of TI was significantly less than that of PI at birth.

Serum multimeric adiponectin levels. Concentrations of adiponectin in cord serum were similar to those in serum of neonates at birth; therefore, we compared adiponectin levels of cord serum and serum levels of PI at corrected term (14). As shown in Table 1, serum concentrations of T-adn, H-adn, $\mathrm{M}-\mathrm{adn}$, and L-adn in PI at birth were significantly less than those in TI. Those of PI at corrected term were similar to those of TI. In PI at corrected term, all of these parameters were significantly higher than those of PI at birth. The ratio of $\mathrm{H}$-adn to T-adn (H/T-adn) of PI at birth was significantly less than those of PI at corrected term and TI. H/T-adn of PI at corrected term was significantly less than that of TI.

Tables $2-4$ show the univariate correlations between T-adn, $\mathrm{H}-\mathrm{adn}, \mathrm{H} / \mathrm{T}-\mathrm{adn}$, and other parameters in PI at birth and at corrected term $(n=46)$ and those in TI. In PI at birth (Table 2 ), serum concentrations of T-adn and $\mathrm{H}$-adn were positively correlated with postmenstrual age, placental weight, body weight, body length, BMI, and Ponderal index $(r=0.31-$ $0.79, p=0.036-0.000)$. H/T-adn was positively correlated
Table 2. Univariate correlations between T-adn, H-adn, H/T-adn, and clinical variables of preterm infants at birth $(\mathrm{n}=46)$

\begin{tabular}{|c|c|c|c|c|c|c|}
\hline \multirow[b]{2}{*}{ Independent variables } & \multicolumn{2}{|c|}{ T-adn } & \multicolumn{2}{|c|}{ H-adn } & \multicolumn{2}{|c|}{ H/T-adn } \\
\hline & $r$ & $p$ & $r$ & $p$ & $r$ & $p$ \\
\hline Postmenstrual age (wk) & 0.75 & 0.000 & 0.75 & 0.000 & 0.48 & 0.001 \\
\hline Placental weight (g) & 0.59 & 0.000 & 0.52 & 0.001 & 0.29 & 0.080 \\
\hline Body weight (g) & 0.79 & 0.000 & 0.73 & 0.000 & 0.44 & 0.003 \\
\hline Body length (cm) & 0.75 & 0.000 & 0.66 & 0.000 & 0.39 & 0.009 \\
\hline $\operatorname{BMI}\left(\mathrm{kg} / \mathrm{m}^{2}\right)$ & 0.77 & 0.000 & 0.77 & 0.000 & 0.48 & 0.001 \\
\hline Ponderal index $\left(\mathrm{kg} / \mathrm{m}^{3}\right)$ & 0.31 & 0.036 & 0.47 & 0.001 & 0.36 & 0.018 \\
\hline $\mathrm{SBP}(\mathrm{mm} \mathrm{Hg})$ & 0.21 & 0.196 & 0.23 & 0.175 & 0.13 & 0.444 \\
\hline DBP (mm Hg) & -0.01 & 0.953 & 0.04 & 0.814 & -0.02 & 0.886 \\
\hline Heart rate & -0.02 & 0.898 & -0.09 & 0.590 & -0.02 & 0.889 \\
\hline
\end{tabular}

Bold type indicates significant correlations.

Table 3. Univariate correlations between T-adn, H-adn, H/T-adn, and clinical variables of preterm infants at corrected term $(\mathrm{n}=46)$

\begin{tabular}{|c|c|c|c|c|c|c|}
\hline \multirow[b]{2}{*}{ Independent variables } & \multicolumn{2}{|c|}{ T-adn } & \multicolumn{2}{|c|}{ H-adn } & \multicolumn{2}{|c|}{$\mathrm{H} / \mathrm{T}$-adn } \\
\hline & $r$ & $p$ & $r$ & $p$ & $r$ & $P$ \\
\hline Postmenstrual age (wk) & 0.14 & 0.371 & 0.30 & 0.047 & 0.38 & 0.010 \\
\hline Placental weight (g) & 0.29 & 0.069 & 0.38 & 0.017 & 0.24 & 0.138 \\
\hline Body weight (g) & 0.34 & 0.021 & 0.41 & 0.005 & 0.19 & 0.209 \\
\hline Body length (cm) & 0.26 & 0.085 & 0.36 & 0.014 & 0.26 & 0.086 \\
\hline BMI $\left(\mathrm{kg} / \mathrm{m}^{2}\right)$ & 0.14 & 0.340 & 0.09 & 0.570 & -0.12 & 0.416 \\
\hline Ponderal index $\left(\mathrm{kg} / \mathrm{m}^{3}\right)$ & 0.08 & 0.609 & 0.04 & 0.782 & -0.08 & 0.619 \\
\hline $\mathrm{SBP}(\mathrm{mm} \mathrm{Hg})$ & 0.32 & 0.030 & 0.18 & 0.241 & -0.30 & 0.040 \\
\hline DBP (mm Hg) & 0.13 & 0.379 & 0.06 & 0.684 & -0.15 & 0.311 \\
\hline Heart rate & 0.29 & 0.051 & 0.06 & 0.685 & -0.50 & 0.000 \\
\hline
\end{tabular}

Bold type indicates significant correlations.

with postmenstrual age, body weight, body length, BMI, and Ponderal index $(r=0.36-0.48, p=0.018-0.001)$. No significant correlation was found among T-adn, H-adn, H/Tadn, and other parameters. In PI at corrected term (Table 3), serum concentrations of T-adn were positively correlated with body weight and SBP $(r=0.32-0.34, p=0.03-0.021)$. Serum concentration of $\mathrm{H}$-adn was positively correlated with postmenstrual age, placental weight, body weight, and body 
Table 4. Univariate correlations between T-adn, H-adn, H/T-adn, and clinical variables of term infants $(\mathrm{n}=26)$

\begin{tabular}{|c|c|c|c|c|c|c|}
\hline \multirow[b]{2}{*}{ Independent variables } & \multicolumn{2}{|c|}{ T-adn } & \multicolumn{2}{|c|}{$\mathrm{H}-\mathrm{adn}$} & \multicolumn{2}{|c|}{ H/T-adn } \\
\hline & $r$ & $p$ & $r$ & $p$ & $r$ & $p$ \\
\hline Postmenstrual age (wk) & 0.26 & 0.195 & 0.37 & 0.060 & 0.39 & 0.047 \\
\hline Placental weight $(\mathrm{g})$ & 0.12 & 0.567 & 0.04 & 0.863 & -0.23 & 0.273 \\
\hline Body weight (g) & 0.39 & 0.049 & 0.40 & 0.045 & 0.10 & 0.625 \\
\hline Body length $(\mathrm{cm})$ & 0.18 & 0.383 & 0.17 & 0.409 & 0.01 & 0.962 \\
\hline $\operatorname{BMI}\left(\mathrm{kg} / \mathrm{m}^{2}\right)$ & 0.43 & 0.027 & 0.46 & 0.017 & 0.18 & 0.369 \\
\hline Ponderal index $\left(\mathrm{kg} / \mathrm{m}^{3}\right)$ & 0.31 & 0.119 & 0.36 & 0.069 & 0.21 & 0.296 \\
\hline $\mathrm{SBP}(\mathrm{mm} \mathrm{Hg})$ & 0.39 & 0.047 & 0.44 & 0.023 & 0.24 & 0.236 \\
\hline DBP (mm Hg) & 0.45 & 0.022 & 0.52 & 0.007 & 0.31 & 0.125 \\
\hline Heart rate & 0.09 & 0.674 & 0.07 & 0.736 & -0.03 & 0.871 \\
\hline
\end{tabular}

Bold type indicates significant correlations.

Table 5. Stepwise multiple regression models for predicting T-adn, $H$-adn, and $H / T-a d n$

\begin{tabular}{|c|c|c|c|}
\hline & $\begin{array}{l}\text { Selected independent } \\
\text { parameters }\end{array}$ & $r^{2}$ & $p$ \\
\hline \multicolumn{4}{|c|}{ Preterm infants at birth $(n=46)$} \\
\hline T-adn & Body weight & 0.62 & 0.000 \\
\hline H-adn & BMI & 0.59 & 0.000 \\
\hline H/T-adn & Postmenstrual age & 0.23 & 0.001 \\
\hline \multicolumn{4}{|c|}{ Preterm infants at corrected term $(n=46)$} \\
\hline T-adn & SBP, placental weight & 0.22 & 0.011 \\
\hline H-adn & Body weight & 0.17 & 0.005 \\
\hline H/T-adn & HR, body weight & 0.34 & 0.000 \\
\hline \multicolumn{4}{|c|}{ Term infants at birth $(n=26)$} \\
\hline T-adn & DBP & 0.20 & 0.022 \\
\hline H-adn & DBP & 0.27 & 0.007 \\
\hline H/T-adn & $\begin{array}{l}\text { Postmenstrual age, } \\
\text { placental weight }\end{array}$ & 0.33 & 0.011 \\
\hline
\end{tabular}

length $(r=0.30-0.41, p=0.047-0.005)$. H/T-adn was positively correlated with postmenstrual age $(r=0.38, p=$ 0.010). SBP and HR showed significant negative correlations with $\mathrm{H} / \mathrm{T}-\operatorname{adn}(r=-0.30$ to $-0.50, p=0.04-0.000)$. No significant correlation was found among T-adn, H-adn, H/Tadn, and other parameters. In TI (Table 4), serum concentration of T-adn was positively correlated with body weight, BMI, SBP, and DBP ( $r=0.39-0.45, p=0.049-0.022)$, as well as $\mathrm{H}$-adn was positively correlated with body weight, BMI, SBP, and DBP ( $r=0.40-0.52, p=0.045-0.007)$. $\mathrm{H} / \mathrm{T}$-adn was positively correlated with postmenstrual age $(r=0.39, p=0.047)$. No significant correlation was found among T-adn, H-adn, H/T-adn, and other parameters.

Because each of the above parameters can potentially contribute directly to the regulation of serum multimeric adiponectin levels, we performed stepwise multiple regression analysis with $\mathrm{T}$-adn, $\mathrm{H}$-adn, and $\mathrm{H} / \mathrm{T}$-adn as the dependent variables and postmenstrual age, placental weight, body weight, body length, BMI, SBP, DBP, and HR as the independent variables (Table 5). In PI at birth, the predictors of T-adn, H-adn, and H/T-adn were body weight, BMI, and postmenstrual age, respectively. In PI at corrected term, the major predictors of T-adn were SBP and placental weight. The predictor of $\mathrm{H}$-adn was body weight. HR and body weight were selected as the predictors of H/T-adn. In TI at birth, the predictor of T- and $\mathrm{H}$-adn was DBP. Postmenstrual age and placental weight were selected as the predictors of $\mathrm{H} / \mathrm{T}$-adn.

\section{DISCUSSION}

In the present study, we showed that i) the serum concentrations of T-adn and $\mathrm{H}$-adn in PI at corrected term were three times higher than those in PI at birth, whereas serum concentrations of T-adn and $\mathrm{H}$-adn of PI at corrected term were similar to those of TI; ii) the H/T-adn of TI and PI at corrected term were significantly greater than that of PI at birth, but that of PI at corrected term was still significantly less than that of $\mathrm{TI}$; and iii) the factors contributing to serum concentrations of $\mathrm{T}$ - and $\mathrm{H}$-adn differed among the three groups.

A recent study reported that concentrations of adiponectin in fetal circulation shows a 20 -fold increase between week 24 of gestation and term, and serum concentrations of adiponectin were positively associated with body weight in PIs (16). In addition, Kotani et al. (14) reported that the cord serum adiponectin concentrations in full-term neonates are higher than serum adiponectin concentrations in adults $(22.4 \mu \mathrm{g} / \mathrm{mL}$ versus $8.2 \mu \mathrm{g} /$ $\mathrm{mL}$ ) and are correlated positively with fat mass-related parameters (birth weight, BMI, and leptin concentration). In the present study, serum concentrations of T- and $\mathrm{H}$-adn increased by $2.5-$ 3 -fold between 32 and $38 \mathrm{wk}$ of gestation, and their levels were also higher than those reported in schoolchildren $(2,7,17)$. These data suggest that fetal development during late pregnancy contributes to serum concentrations of $\mathrm{T}$ - and $\mathrm{H}$-adn, and that the relationship between adiposity and adiponectin levels in neonates may differ from that in adults and children. Interestingly, Inami et al. (18) recently reported a positive association between serum concentrations of adiponectin and body weight in TIs at birth, but no association was found at 1 mo of age. To date, some roles of adipocytokines such as IL- 6 and TNF- $\alpha$ have been highlighted in the inverse correlation between adiponectin and body weight in adults (19). It has been shown that IL-6 and TNF- $\alpha$ reduce the secretion of adiponectin from adipocytes (20). Some types of inflammation in adipose tissue might contribute to the inverse correlation between adiponectin and adiposity. The rapid increase in fetal body weight during late pregnancy and the perinatal period is a physiologic development; thus, it is reasonable to consider that an increase in adipose tissue at this stage may not be accompanied by hypertrophy of adipocytes and the inflammation in adipose tissue. This may also cause the higher levels of adiponectin and positive correlations between adiponectin and body weight observed during the perinatal period. With respect to the higher concentrations of adiponectin in PI at corrected term and TI, Kim et al. (21) reported that serum levels of adiponectin are a starvation signal released by adipocytes, and that normalsized adipocytes do not induce insulin resistance, even in obese status. It is well known that development of adipose tissue is greatest in perinatal period (22). A recent report of Pinar et al. (23) showed that the high concentrations of $\mathrm{H}$-adn found in the fetus were associated with higher insulin sensitivity. Thus, high levels of adiponectin in the perinatal period may reflect rapid growth during this period.

As shown in Table 2, T-adn, $\mathrm{H}$-adn, and $\mathrm{H} / \mathrm{T}$-adn were significantly associated with adiposity-related parameters (body weight, BMI, and Ponderal index) in PI at birth. However, these associations were weakened in PI at corrected term and TI (Tables 3 and 4). Different from PI at birth, hemodynamic 
parameters (blood pressure and/ or HR) were significantly associated with T-adn and $\mathrm{H} / \mathrm{T}$-adn in PI at corrected term, and with T-adn and H-adn in TI (Tables 3-5). It is of interest that expression of adiponectin in fetus was pronounced in vascular endothelium, thereby suggesting that in addition to adipose tissues, vascular endothelial cells may contribute to fetal adiponectin levels (23). It is plausible that hemodynamic parameters affect the status of vascular endothelium and lead to significant correlation of these to adiponectin in PI at corrected term and TI. Taken together, our data suggest that vascular endothelium might contribute to serum concentrations of $\mathrm{T}$-adn and $\mathrm{H}$-adn during late pregnancy. However, in PI at corrected term, hemodynamic parameters were not associated with $\mathrm{H}$-adn. SBP and HR were inversely associated with $\mathrm{H} / \mathrm{T}$-adn. These results indicate that in PI at corrected term, ex-utero factors may influence adipose tissue development and contribution of vascular endothelium to adiponectin status. Placental weight was significantly correlated with T-adn and $\mathrm{H}$-adn in PI at birth (Table 2). Serum concentrations of $\mathrm{H}$-adn in PI at corrected term were still positively associated with placental weight (Table 3). A recent report showed that the adiponectin concentration in umbilical cord serum is positively associated with the weight ratio of fetus to placenta (24). In contrast, Pinar et al. proved the absence of endogenous placental adiponectin and showed its exclusive production by fetal tissues. All of these findings indicate that effect of placental weight on adiponectin status may be indirect effects; however, further studies are needed to clarify this.

Wang et al. (25) showed that ectopic fat accumulation occurred even in nonobese mice, which showed hyperinsulinemia, insulin resistance, and hypertrophy of adipocytes because of subnormal adipocyte storage capacity, whereas Ruderman et al. (26) reported metabolically obese, normal-weight patients. These previous studies, combined with the report by Kim et al. (21) strongly suggest that in neonates, normal development of adipose tissue is important to prevent future development of cardiometabolic diseases such as diabetes, hypertension, and dyslipidemia. Subnormal development of adipose tissue in neonates may reduce the number of adipocytes. If our notion is valid, the limited number of adipocytes in subjects with low birth weight may induce hypertrophy of adipocytes more readily than in subjects with normal birth weight. As a result, these subjects may be at high risk for cardiometabolic diseases in the future, even if normal weight is maintained. Ibanez et al. (27) recently reported that children born small for gestational age tend to be viscerally adipose with hypoadiponectinemia, even if they are not overweight. This may support our hypothesis.

In conclusion, our present data suggest that postnatal growth accompanying adiposity similar to TI may be important for premature and/or low birth weight infant to prevent future development of cardiometabolic diseases. Monitoring of $\mathrm{T}$ - and $\mathrm{H}$-adn could be useful to evaluate the maturation of adipose tissue in PIs.

Acknowledgments. We are grateful to the medical and nursing staffs of the neonatal intensive care unit at The Ryukyu University Hospital for their help for this study.

\section{REFERENCES}

1. Irving RJ, Belton NR, Elton RA, Walker BR 2000 Adult cardiovascular risk factors in premature babies. Lancet 355:2135-2136

2. Kaneshi T, Yoshida T, Ohshiro T, Nagasaki H, Asato Y, Ohta T 2007 Birthweight and risk factors for cardiovascular diseases in Japanese schoolchildren. Pediatr Int 49:138-143

3. Barker DJ 2004 The developmental origins of adult disease. J Am Coll Nutr 23:588S-595S

4. Arita Y, Kihara S, Ouchi N, Takahashi M, Maeda K, Miyagawa J, Hotta K, Shimomura I, Nakamura T, Miyaoka K, Kuriyama H, Nishida M, Yamashita S, Okubo K, Matsubara K, Muraguchi M, Ohmoto Y, Funahashi T, Matsuzawa Y 1999 Paradoxical decrease of an adipose-specific protein, adiponectin, in obesity. Biochem Biophys Res Commun 257:79-83

5. Weyer C, Funahashi T, Tanaka S, Hotta K, Matsuzawa Y, Pratley RE, Tataranni PA 2001 Hypoadiponectinemia in obesity and type 2 diabetes: close association with insulin resistance and hyperinsulinemia. J Clin Endocrinol Metab 86:1930-1935

6. Kumada M, Kihara S, Sumitsuji S, Kawamoto T, Matsumoto S, Ouchi N, Arita Y, Okamoto Y, Shimomura I, Hiraoka H, Nakamura T, Funahashi T, Matsuzawa Y 2003 Association of hypoadiponectinemia with coronary artery disease in men. Arterioscler Thromb Vasc Biol 23:85-89

7. Yoshida T, Kaneshi T, Shimabukuro T, Sunagawa M, Ohta T 2006 Serum C-reactive protein and its relation to cardiovascular risk factors and adipocytokines in Japanese children. J Clin Endocrinol Metab 91:2133-2137

8. Pajvani UB, Du X, Combs TP, Berg AH, Rajala MW, Schulthess T, Engel J, Brownlee M, Scherer PE 2003 Structure-function studies of the adipocyte-secreted hormone Acrp30/adiponectin. Implications for metabolic regulation and bioactivity. J Biol Chem 278:9073-9085

9. Hug C, Wang J, Ahmad NS, Bogan JS, Tsao T-S, Lodish HF 2004 T-cadherin is a receptor for hexameric and high-molecular-weight forms of Acrp30/adiponectin. Proc Natl Acad Sci USA 101:10308-10313

10. Hada Y, Yamauchi T, Waki H, Tsuchida A, Hara K, Yago H, Miyazaki O, Ebinuma H, Kadowaki T 2007 Selective purification and characterization of adiponectin multimer species from human plasma. Biochem Biophys Res Commun 356:487-493

11. Waki H, Yamauchi T, Kamon J, Ito Y, Uchida S, Kita S, Hara K, Hada Y, Vasseur F, Froguel P, Kimura S, Nagai R, Kadowaki T 2003 Impaired multimerization of human adiponectin mutants associated with diabetes. Molecular structure and multimer formation of adiponectin. J Biol Chem 278:40352-40363

12. Aso Y, Yamamoto R, Wakabayashi S, Uchida T, Takayanagi K, Takebayashi K, Okuno T, Inoue T, Node K, Tobe T, Inukai T, Nakano Y 2006 Comparison of serum high-molecular weight (HMW) adiponectin with total adiponectin concentrations in type 2 diabetic patients with coronary artery disease using a novel enzyme-linked immunosorbent assay to detect HMW adiponectin. Diabetes 55:1954-1960

13. Nemet D, Wang P, Funahashi T, Matsuzawa Y, Tanaka S, Engelman L, Cooper DM 2003 Adipocytokines, body composition, and fitness in children. Pediatr Res 53:148-152

14. Kotani Y, Yokota I, Kitamura S, Matsuda J, Naito E, Kuroda Y 2004 Plasma adiponectin levels in newborns are higher than those in adults and positively correlated with birth weight. Clin Endocrinol (Oxf) 61:418-423

15. Ebinuma H, Miyazaki O, Yago H, Hara K, Yamauchi T, Kadowaki T 2006 A novel ELISA system for selective measurement of human adiponectin multimers by using proteases. Clin Chim Acta 372:47-53

16. Kajantie E, Hytinantti T, Hovi P, Andersson S 2004 Cord plasma adiponectin: A 20-fold rise between 24 weeks gestation and term. J Clin Endocrinol Metab 89:4031-4036

17. Araki S, Dobashi K, Kubo K, Asayama K, Shirahata A 2006 High molecular weight, rather than total, adiponectin levels better reflect metabolic abnormalities associated with childhood obesity. J Clin Endocrinol Metab 91:5113-5116

18. Inami I, Okada T, Fujita H, Makimoto M, Hosono S, Minato M, Takahashi S, Harada $\mathrm{K}$, Yamamoto T 2007 Impact of serum adiponectin concentration on birth size and early postnatal growth. Pediatr Res 61:604-606

19. Kern PA, Di Gregorio GB, Lu T, Rassouli N, Ranganathan G 2003 Adiponectin expression from human adipose tissue: relation to obesity, insulin resistance, and tumor necrosis factor-alpha expression. Diabetes 52:1779-1785

20. Engeli S, Feldpausch M, Gorzelniak K, Hartwig F, Heintze U, Janke J, Mohlig M, Pfeiffer AF, Luft FC, Sharma AM 2003 Association between adiponectin and mediators of inflammation in obese women. Diabetes 52:942-947

21. Kim JY, van de Wall E, Laplante M, Azzara A, Trujillo ME, Hofmann SM, Schraw T, Durand JL, Li H, Li G, Jelicks LA, Mehler MF, Hui DY, Deshaies Y, Shulman GI, Schwartz GJ, Scherer PE 2007 Obesity-associated improvements in metabolic profile through expansion of adipose tissue. J Clin Invest 117:2621-2637

22. Whitaker RC, Dietz WH 1998 The role of the prenatal environment in the development of obesity. J Pediatr 132:768-776

23. Pinar H, Basu S, Hotmire K, Laffineuse L, Presley L, Carpenter M, Catalano PM, Hauguel-de Mouzon S 2008 High molecular mass multimer complexes and vascular expression contribute to high adiponectin in the fetus. J Clin Endocrinol Metab 93:2885-2890

24. Kadowaki K, Waguri M, Nakanishi I, Miyashita Y, Nakayama M, Suehara N, Funahashi T, Shimomura I, Fujita T 2006 Adiponectin concentration in umbilical cord serum is positively associated with the weight ratio of fetus to placenta. J Clin Endocrinol Metab 91:5090-5094

25. Wang MY, Grayburn P, Chen S, Ravazzola M, Orci L, Unger RH 2008 Adipogenic capacity and the susceptibility to type 2 diabetes and metabolic syndrome. Proc Natl Acad Sci USA 105:6139-6144

26. Ruderman N, Chisholm D, Pi-Sunyer X, Schneider S 1998 The metabolically obese, normal-weight individual revisited. Diabetes 47:699-713

27. Ibáñez L, Lopez-Bermejo A, Suárez L, Marcos MV, Díaz M, de Zegher F 2008 Visceral adiposity without overweight in children born small for gestational age. J Clin Endocrinol Metab 93:2079-2083 